Research Article

\title{
Low Dose of Lipopolysaccharide Pretreatment Preventing Subsequent Endotoxin-Induced Uveitis Is Associated with PI3K/AKT Pathway
}

\author{
Nan Zhang, Shuo Yu, Xinli Liu, and Hong Lu \\ Department of Ophthalmology, Beijing Chao Yang Hospital, Capital Medical University, No. 8 South Gongren Tiyuchang Road, \\ Chao Yang District, Beijing 100020, China
}

Correspondence should be addressed to Hong Lu; honglucmu@outlook.com

Received 20 January 2017; Revised 29 May 2017; Accepted 1 June 2017; Published 18 July 2017

Academic Editor: Mario Clerici

Copyright () 2017 Nan Zhang et al. This is an open access article distributed under the Creative Commons Attribution License, which permits unrestricted use, distribution, and reproduction in any medium, provided the original work is properly cited.

Purpose. To explore the effects of LPS pretreatment on endotoxin-induced uveitis and PI3K/AKT pathway. Methods. Endotoxininduced uveitis was induced by a single subcutaneous injection of $200 \mu \mathrm{g}$ LPS. For the endotoxin tolerance group, induction of EIU was preceded by daily subcutaneous injection of $0.1 \mathrm{mg} / \mathrm{kg}$ LPS for five days. Clinical scores were graded at $24 \mathrm{~h}$ after EIU under a slit lamp microscope. HE stain was performed to observe the histopathology. Aqueous humor TNF- $\alpha$ concentration was quantified with enzyme-linked immunosorbent assay. The expressions of PI3K and AKT were detected through Western blot analyses, and the activation of AKT was detected through immunofluorescence study. Results. Endotoxin tolerance produced suppressive effects by significantly reducing the inflammatory reaction of anterior segment of the rats as measured by slit lamp and histopathology. Low dose of LPS pretreatment significantly reduced TNF- $\alpha$ concentrations and the expressions of PI3K and AKT. Furthermore, the activation of AKT was also inhibited. Conclusions. LPS pretreatment can ameliorate endotoxin-induced uveitis in rats. This protection of endotoxin tolerance against EIU is associated with PI3K/AKT pathway by reducing level of TNF- $\alpha$ in the aqueous humor.

\section{Introduction}

Uveitis is one of the world's major sight-threatening diseases, which mainly affects the iris, ciliary body, and choroid [1]. Lipopolysaccharide (LPS), which is a kind of pathogenassociated molecular patterns (PAMPs), activates the innate immune system through toll-like receptor 4 (TLR4) which is a member of innate immune recognition receptors (PRRs) [2]. Substantial clinical and experimental evidences support the role of LPS in the pathogenesis of immunemediated, noninfectious acute anterior uveitis (AAU), particularly human leukocyte antigen- (HLA-) B27-associated AAU [3]. In terms of LPS-induced inflammatory response, there is an important protective mechanism, which is called endotoxin tolerance that the body or cells preexposed to low dose of LPS are refractory to subsequent LPS challenge [4]. Elucidating the effect of endotoxin tolerance on ocular inflammation and the underlying mechanism are of great importance for the prevention and treatment of uveitis.

Endotoxin-induced uveitis (EIU) with the injection of LPS into certain susceptible strains of rodents inducing an acute and preferential inflammation of the iris and ciliary body is a well-established animal model that closely resembles AAU in humans [5]. The inflammation of the cellular infiltration and protein leakage into the anterior chamber of the eye reaches a maximum $24 \mathrm{~h}$ after LPS injection [6]. Elevated expression of cytokines such as TNF- $\alpha$ and IL-6 has been observed to be concomitant with maximum EIU $[6,7]$. Cytokine levels, especially those of TNF- $\alpha$, can be 
used as a marker for monitoring the inflammation and disease progression.

As is known to us, LPS could activate the PI3K/AKT pathway, which regulates the expressions of cytokine and chemokine, such as TNF- $\alpha$ [8-10], moreover, which is involved in endotoxin tolerance [11]. Furthermore, a study of gene expression microarray analysis revealed that PI3K and AKT play critical roles in HLA-B27-associated AA [12]. We sought to explore the effect of endotoxin tolerance on endotoxin-induced uveitis and evaluate whether endotoxin tolerance could modulate the PI3K/AKT pathway.

\section{Materials and Methods}

2.1. Animals. Inbred male pathogen-free Wistar rats (8-10 weeks old, weighing 180-200 g) were obtained from the Vital River Laboratory Animal Technology Co. Ltd. (Beijing, China) and maintained in an air-conditioned room with $12 \mathrm{~h}$ light $/ 12 \mathrm{~h}$ dark cycles. Food and water were supplied ad libitum. Experiments were conducted in accordance with the Institute for Laboratory Animal Research guidelines (Guide for the Care and Use of Laboratory Animals).

2.2. Reagents. Lipopolysaccharide (V. cholerae, classical Biotype, serotype Ogawa) was provided by the Lanzhou Biological Product Research. Rabbit polyclonal PI3-kinase p $85 \alpha$ antibody and rabbit polyclonal AKT 1/2/3 antibody were purchased from Santa Cruz Biotechnology, CA, USA. AffiniPure Fab Fragment Donkey Anti-Rabbit IgG $(\mathrm{H}+\mathrm{L})$, Alexa Fluor $^{\circledR} 488$ were purchased from Jackson ImmunoResearch Laboratories, Inc., PA. ReverTra Ace qPCR RT Master Mix and SYBR Green Realtime PCR Master Mix were purchased from Toyobo (Shanghai) Biotech, Co., Ltd. Rat TNF- $\alpha$ ELISA kit was purchased from Cusabio.

2.3. Animal Model. Endotoxin-induced uveitis (EIU) was induced by a single subcutaneous injection of $200 \mu \mathrm{g}$ LPS dissolved in $0.1 \mathrm{ml}$ sterile saline $(1 \mathrm{mg} / \mathrm{kg})$ as previously described [13]. All animals were randomly divided into three groups: normal control (NC) group, endotoxin tolerance (ET) group, and endotoxin-induced uveitis (EIU) group. In the ET group, endotoxin tolerance was induced by daily subcutaneous injection of $0.1 \mathrm{mg} / \mathrm{kg}$ LPS for five days [14]. The other two groups of rats were treated with sterilized saline in the same manner as pretreatment. On day 6, the animals in the ET and EIU groups received a single subcutaneous injection of $200 \mu \mathrm{g}$ LPS to induce EIU. The NC group received subcutaneous injection of $0.1 \mathrm{ml}$ sterile saline.

2.4. Clinical Manifestations Scoring. Clinical features of ocular inflammation were evaluated in both eyes at $24 \mathrm{~h}$ after injection of $200 \mu \mathrm{g}$ LPS or vehicle under a slit lamp microscope. Inflammatory signs were recorded. The severity of uveitis was graded according to the scoring system in Table 1 by two observers who were blinded to the treatment groups $[15,16]$.

2.5. Enzyme-Linked Immunosorbent Assay (ELISA). After clinical evaluation, an aqueous humor sample was collected
TABLE 1: Scoring system for clinical evaluation of uveitis.

\begin{tabular}{ll}
\hline Clinical signs & Grade of uveitis (score) \\
\hline Iris hyperemia & 0 \\
Absent & 1 \\
Mild & 2 \\
Moderate & 3 \\
Severe & \\
Pupil & 0 \\
Normal & 1 \\
Miosis & \\
Exudate in anterior chamber & 0 \\
Absent & 1 \\
Small & 2 \\
Large & \\
Hypopyon & \\
Absent & 0 \\
Present & 1 \\
Maximum possible score & 7 \\
\hline
\end{tabular}

from both eyes of all rats using a 30-gauge needle attached to a $1 \mathrm{~mL}$ syringe under a microscope. TNF- $\alpha$ levels in the aqueous humor were assessed with rat TNF- $\alpha$ ELISA kit. All measurements were conducted according to the manufacturer's instructions.

2.6. Histopathological Examination. For histopathologic analysis, the rats were killed 24 hours after the last injection of LPS or vehicle; their intact eyes were enucleated and placed in $10 \%$ neutral buffered formalin solution for $24 \mathrm{~h}$. The eye specimens were dehydrated in a graded ethanol series and embedded in paraffin. Sagittal sections (4-5 $\mu \mathrm{m}$ thick), cut near the optic nerve head, were stained with hematoxylin and eosin. As previously described [16, 17], anterior chamber tissues were scored for severity of inflammation as follows: grade $0=$ normal tissue; grade $1+=$ dilated iris vessels and thickened iris stroma with exudate, protein, and/or a few scattered inflammatory cells in the anterior chamber; grade $2+=$ infiltration of inflammatory cells into the stroma of the iris and/or ciliary body with a moderate number of inflammatory cells within the anterior chamber; grade $3+=$ heavy infiltration of inflammatory cells within the iris stroma, ciliary body, and the anterior chamber; and grade $4+=$ heavy exudation of cells, dense protein aggregation in the anterior chamber, and in inflammatory cell deposits on the corneal endothelium. Histopathologic analyses were performed by a single pathologist blinded to treatment history.

2.7. Western Blot. Each ICB sample was harvested, rinsed with PBS, and lysed. A total of $40 \mu \mathrm{g}$ of protein per sample was separated by SDS-polyacrylamide gel electrophoresis, incubated with anti-PI3-kinase p85 $\alpha$ (Santa Cruz Biotechnology) at a dilution of $1: 1000$ or anti-AKT1/2/3 (Santa Cruz Biotechnology) at a dilution of $1: 500$. Signal was visualized after secondary antibody staining (donkey antirabbit IgG antibody, 1:4000). All values were normalized 


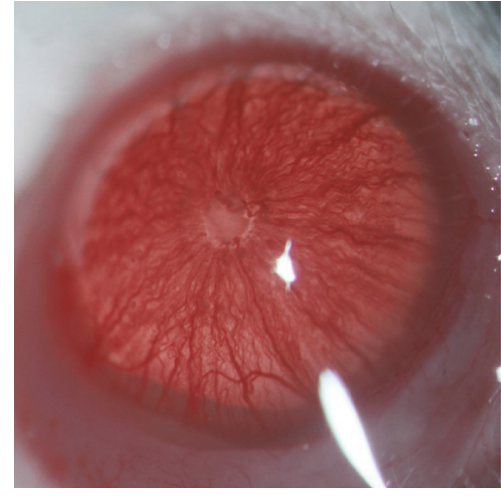

(a)

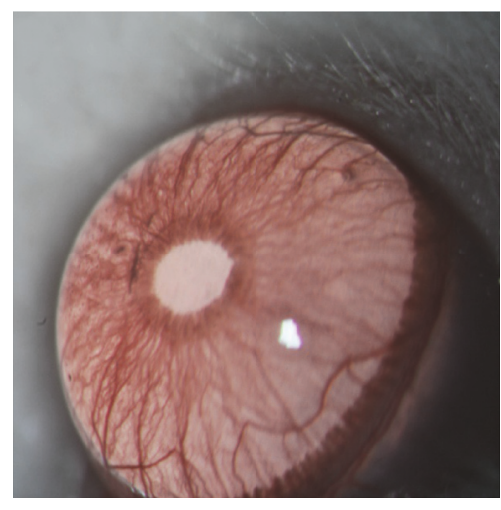

(c)

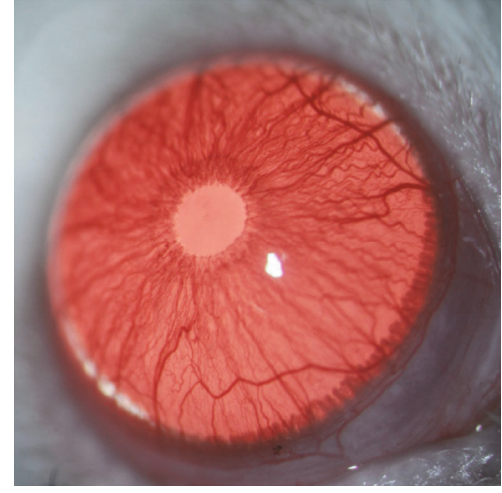

(b)

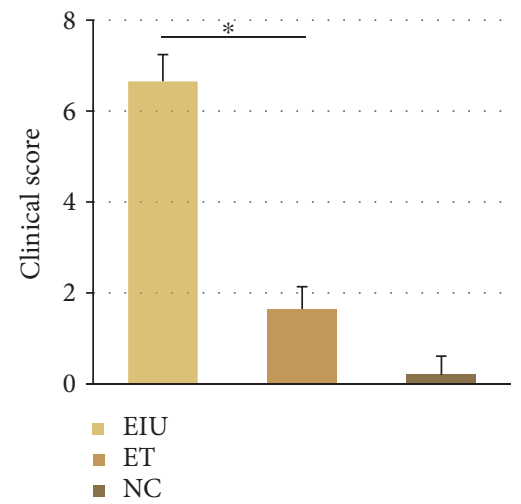

(d)

FIgure 1: (a) Occlusion of pupil and fibrinous membrane was observed in the EIU group at $24 \mathrm{~h}$ after $200 \mu \mathrm{g}$ LPS injection. (b) Slight iris blood vessel dilatation could be found in the ET group at $24 \mathrm{~h}$ after $200 \mu \mathrm{g}$ LPS injection. (c) No ocular inflammatory signs were observed in rats of the NC group. (d) All data shown as mean \pm standard deviation $\left(n=20\right.$, eye). ${ }^{*} p<0.05$ significantly different from EIU group.

with $\beta$-actin as loading control. Each sample was collected from two rats (4 eyes).

2.8. Immunofluorescence. Eye specimens were fixed in $4 \%$ paraformaldehyde for 1-2 $\mathrm{h}$ after being enucleated. The ICB was dissected into four segments; each prepared tissue was permeabilized with $0.3 \%$ Triton X-100 for $30 \mathrm{~min}$ at room temperature and then washed three times with PBS. After blocking nonspecific binding with $3 \%$ bovine serum albumin (BSA)/PBS, the tissues were incubated with rabbit polyclonal PI3-kinase p85 $\alpha$ antibody (1:100 in 3\% BSA/PBS) and rabbit polyclonal AKT $1 / 2 / 3$ antibody (1:100 in $3 \%$ BSA/PBS), respectively, overnight at $4^{\circ} \mathrm{C}$. Excessive antibodies were removed by washing with PBS, three times. The tissues were incubated with AffiniPure Fab Fragment Donkey AntiRabbit IgG $(\mathrm{H}+\mathrm{L})$, Alexa Fluor 488 (1:200 in PBS), for $2 \mathrm{~h}$, protected from light at room temperature. After three washes with PBS for 10 min each, the slides were mounted in Antifade Solution. The negative controls were included replacing the first or second primary antibody or both antibodies with species- and isotype-matched irrelevant antibodies. Blank controls were included replacing the first or second primary antibody with PBS.

The slides were examined under a fluorescence microscope (Leica DM-4000B; Leica, Wetzlar, Germany). The images were captured, and the pairs of images were superimposed for colocalization analysis using imagemanagement software (Adobe Photoshop CS3. 10.0; Adobe Systems, Mountain View, CA).

2.9. Statistical Analysis. Quantitative data were analyzed with one-way analysis of variance (ANOVA) (SPSS 19.0; SPSS Inc., Chicago, IL). Values of $p<0.05$ were considered statistically significant.

\section{Results}

3.1. Endotoxin Tolerance Attenuates EIU. At $24 \mathrm{~h}$ after injection of $200 \mu \mathrm{g}$ LPS or vehicle, the EIU group showed typical signs mimicking human uveitis, including ciliary congestion, iris blood vessel dilatation, pupil occlusion, and fibrinous membrane formation (Figure 1(a)). Low dose of LPS pretreatment (ET group) significantly attenuated iris blood vessel dilatation, pupil occlusion, and fibrinous membrane formation; just slight iris blood vessel dilatation could be found in the ET group, small amounts of exudate occasionally (Figure 1(b)). No ocular inflammatory signs were observed in rats of the blank control group (Figure 1(c)). The clinical score of the ET group was $1.65 \pm 0.49$, significantly lower than that of the EIU group $(6.65 \pm 0.59$, $p<0.05$ ) (Figure 1(d)). 


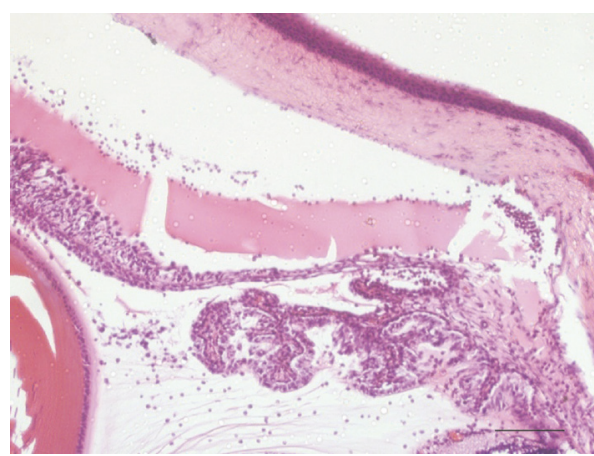

(a)

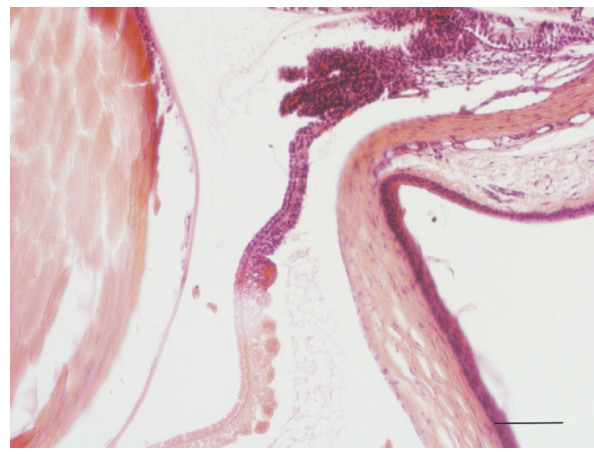

(c)

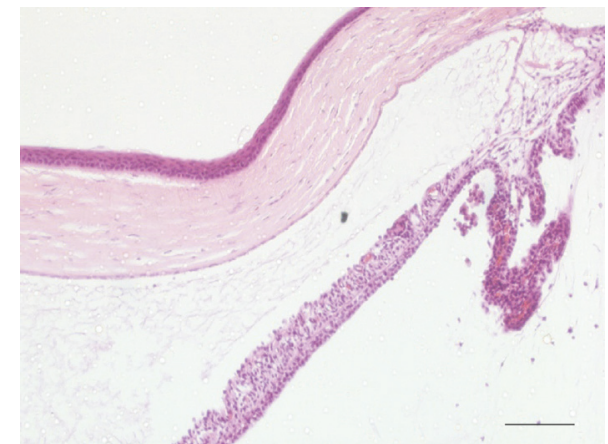

(b)

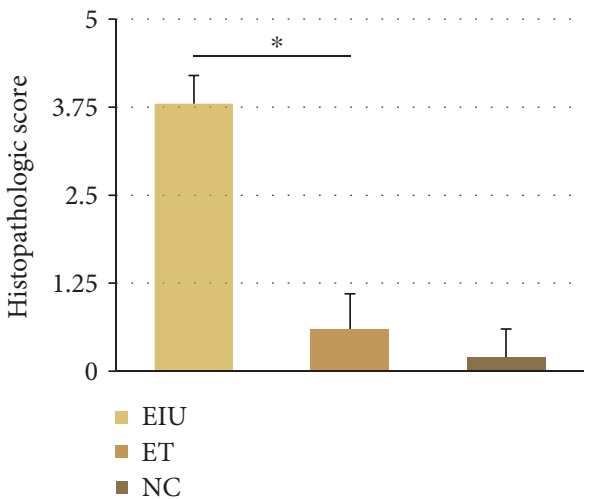

(d)

Figure 2: (a) The EIU group: heavy infiltration of inflammatory cells within the iris stroma, ciliary body, and the anterior chamber and inflammatory cells deposited on the corneal endothelium. (b) The ET group: only a few scattered inflammatory cells. (c) The NC group: no infiltrating cells. Scale bars: $200 \mu \mathrm{m}$. (d) All data shown as mean \pm standard deviation $\left(n=20\right.$, eye). ${ }^{*} p<0.05$ significantly different from EIU group.

3.2. Histopathological Changes. HE staining results were in accordance with clinical features of ocular inflammation. In the EIU group, heavy infiltration of inflammatory cells within the iris stroma, ciliary body, and the anterior chamber and inflammatory cells depositing on the corneal endothelium were observed (Figure 2(a)). Inflammatory cellular infiltration was significantly reduced by low dose of LPS pretreatment in the ET group (Figure 2(b)). The histopathologic score of the ET group was $0.6 \pm 0.5$, significantly lower than that of the EIU group $(3.8 \pm 0.4, p<0.05)$ (Figure $1(\mathrm{~d}))$.

3.3. The Levels of Cytokines in the Aqueous Humor during Endotoxin-Induced Uveitis in Wistar Rats. As shown in Figure 3, the aqueous TNF- $\alpha$ concentration in the EIU group $(30.96 \pm 1.55 \mathrm{pg} / \mathrm{ml})$ was significantly higher than that of the NC group $(10.32 \pm 0.61 \mathrm{pg} / \mathrm{ml})$. However, the level of TNF$\alpha$ was significantly reduced in the ET group when dose of LPS pretreatment $(10.58 \pm 0.67 \mathrm{pg} / \mathrm{ml})$ is low.

3.4. Effects of Endotoxin Tolerance on the Expression of PI3K and $A K T$ in the Iris-Ciliary Body. Western blot was performed to detect the expression of PI3K and AKT. As shown in Figure 4, the protein levels of PI3K and AKT in the ET group decreased significantly compared with the EIU group. Moreover, the levels also decreased significantly compared with the NC group.

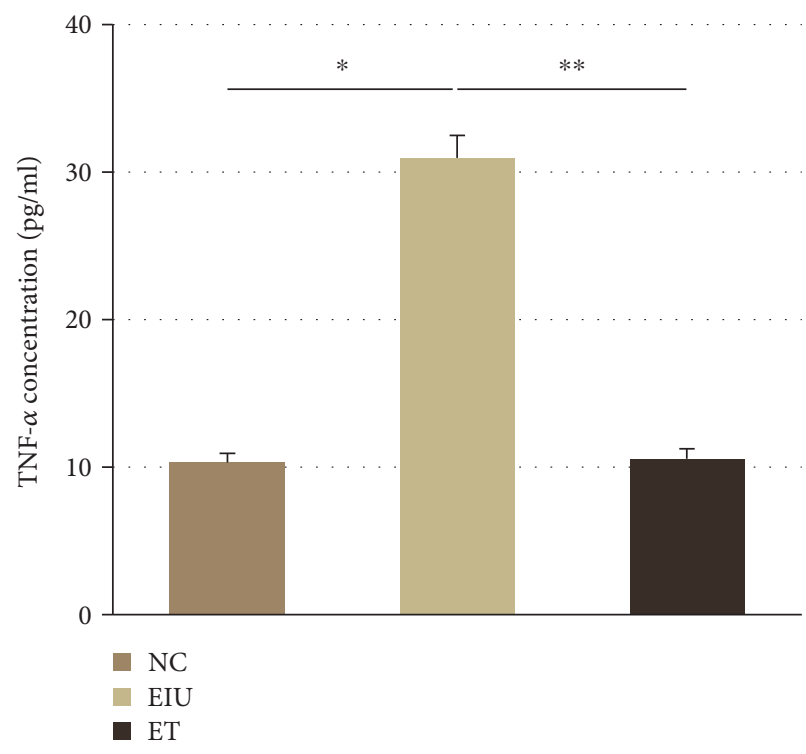

Figure 3: Expression of TNF- $\alpha$ in aqueous humor. All data shown as mean \pm standard deviation $(n=10) .{ }^{*} p<0.05$ the EIU group versus the $\mathrm{NC}$ group; ${ }^{* *} p<0.05$ the ET group versus the EIU group.

3.5. Immunofluorescence Analysis of the AKT Activation. Without LPS stimulation, AKT distributed over cytoplasm (Figure 5(a)). After $200 \mu \mathrm{g}$ LPS stimulation, AKT transferred 


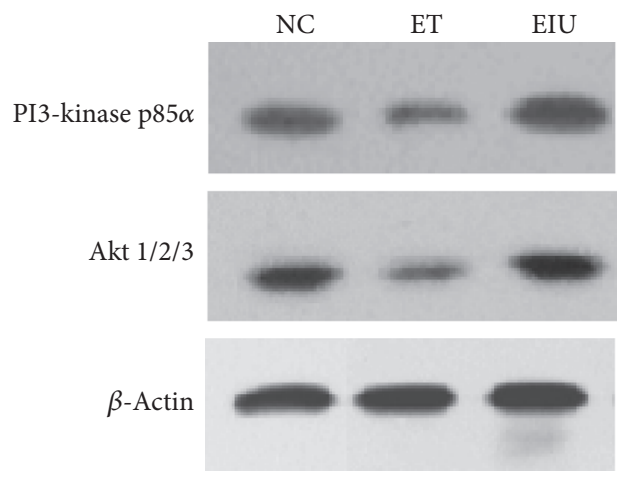

PI3-kinase p85 $\alpha / \beta$-actin

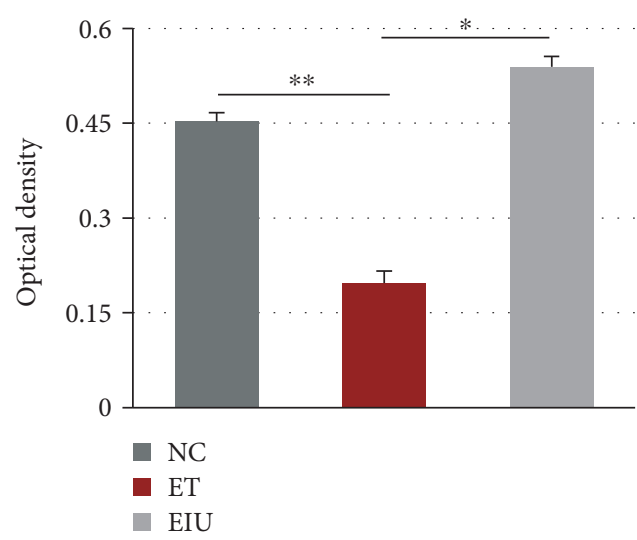

Akt $1 / 2 / 3 / \beta$-actin

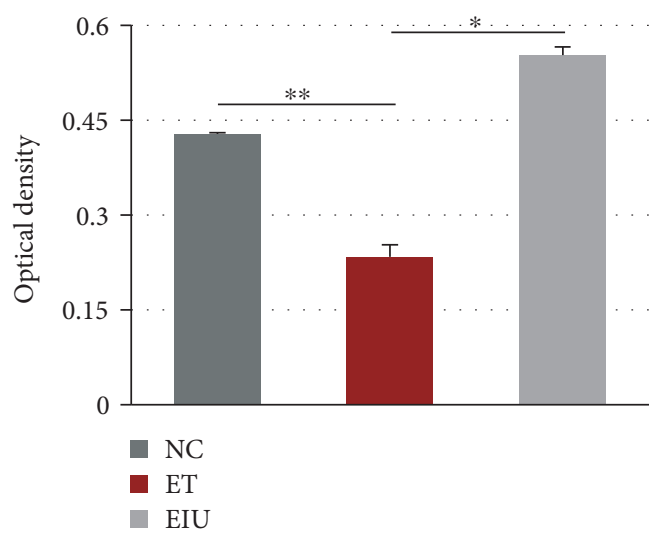

FIgURe 4: Western blot analysis and protein levels of PI3K and AKT. ${ }^{*} p<0.05$ the ET group versus the EIU group; ${ }^{* *} p<0.05$ the ET group versus the NC group. Data represent the mean \pm SD from ten independent experiments.

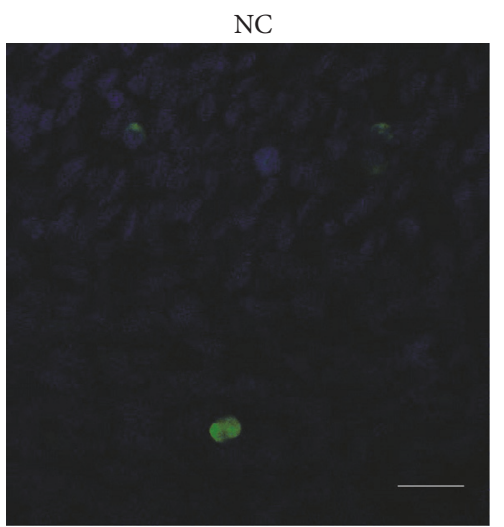

(a)

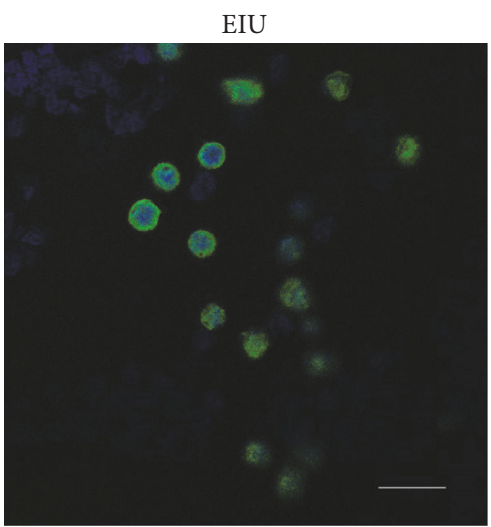

(b)

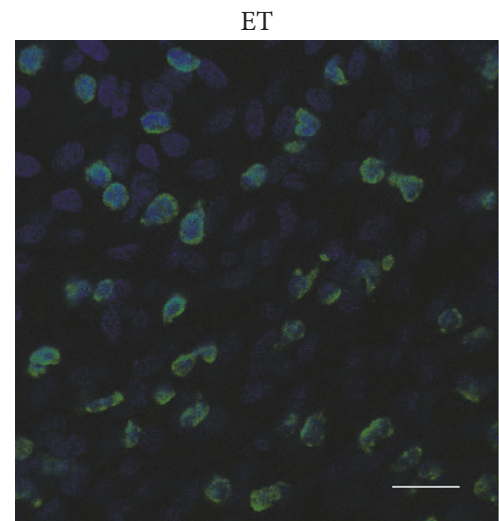

(c)

FIgURE 5: In the NC group, AKT distributed over cytoplasm (a). In the EIU group, AKT transferred from cytoplasm to cell membrane (b). In the ET group, translocation of AKT was inhibited partially (c). Scale bars: $50 \mu \mathrm{m}$.

from cytoplasm to cell membrane, representing activation of AKT (Figure 5(b)). However, low dose of LPS pretreatment inhibited translocation of AKT partially (Figure 5(c)).

\section{Discussion}

As a potent inflammatory stimulant, high doses of LPS lead to systemic inflammatory response syndrome and death
[18]. However, if the body was given a small dose of LPS stimulation in advance, the body showed low or no response to the subsequent large dose or lethal dose of LPS stimulation. This important protective mechanism is called endotoxin tolerance [4].

To elucidate the effect of endotoxin tolerance on ocular inflammation, endotoxin-induced uveitis with that a wellestablished animal model of AAU was used in this study. 
The Wistar rats in the EIU group showed typical signs mimicking human uveitis, including ciliary congestion, iris blood vessel dilatation, pupil occlusion, and fibrinous membrane formation. However, the inflammatory reaction of anterior segment of the rats significantly decreased in the ET group as measured by slit lamp assessment and histopathology. These results demonstrate that endotoxin tolerance which was induced by low dose of LPS pretreatment could successfully suppress the ocular inflammation in the EIU model.

Further, these findings suggest that endotoxin tolerance is of great potential in the treatment and prevention of uveitis. However, the molecular mechanisms underlying the induction of endotoxin tolerance are not yet fully understood. Hoekzema et al. [15] demonstrated that repeated injection of endotoxin resulted in no detectable IL- 6 in the aqueous humor and the absence of uveitis. Mashimo et al. [19] indicated that continuous high expression of IL-10 in the eye plays significant role in the mechanism of endotoxin tolerance in a rat model of EIU. In this study, TNF- $\alpha$ levels in the aqueous humor during endotoxin-induced uveitis in rats were investigated to further clarify the anti-inflammatory effect of endotoxin tolerance and explore the role of cytokines in this protective mechanism. Consistent with the literatures $[6,20-22]$, elevated TNF- $\alpha$ was observed in the EIU group, and the TNF- $\alpha$ levels were significantly reduced by low dose of LPS pretreatment in ET group. This result strongly confirmed the anti-inflammatory effect of endotoxin tolerance, which is consistent with the slit lamp assessment and histopathology. Furthermore, the result suggests that TNF- $\alpha$ as well as IL-6 and IL-10 is closely involved in the protective mechanism of endotoxin tolerance. However, the upstream mechanism remains unclear. As mentioned earlier, the $\mathrm{PI} 3 \mathrm{~K} / \mathrm{AKT}$ pathway plays an important role in regulating LPS-induced cytokine such as TNF- $\alpha$ expression. Therefore, we next evaluated whether endotoxin tolerance could modulate the PI3K/AKT pathway.

Similar to many protein kinases, AKT has a specific AH/ $\mathrm{PH}$ domain which mediates the interaction between signaling molecules. PI3K generates phosphoinositide-3,4,5-triphosphate (PIP3) that can bind to the AH/PH domain of AKT in response to upstream signals, resulting in AKT translocating to the plasma membrane and being activated [23]. Thus, both the expressions of PI3K and AKT in ICB of the EIU rats and activation of $\mathrm{AKT}$ were detected. In this work, the protein levels of PI3K and AKT in the ET group decreased significantly compared with the EIU group, which were associated with the inflammatory outcome of the rats. Moreover, the levels in the ET group were even lower than those in the NC group, which indicated that the expression of PI3K and AKT was suppressed when dose of LPS pretreatment is low. Furthermore, according to the immunofluorescence, the activation of AKT was also suppressed in the ET group. Taken together, both low expressions of PI3K/AKT and inhibition of AKT activation contribute to the induction of endotoxin tolerance.

In summary, the present study demonstrates that low dose of LPS pretreatment could prevent subsequent endotoxininduced uveitis by reducing the expression of TNF- $\alpha$ in the aqueous humor, and this protective effect of endotoxin tolerance is associated with PI3K/AKT pathway. Furthermore, in virtue of the critical roles of PI3K and AKT in HLA-B27associated acute anterior uveitis, endotoxin tolerance holds promise for the prevention and treatment of this sightthreatening diseases.

\section{Conflicts of Interest}

The authors declare that they have no conflicts of interest.

\section{Acknowledgments}

This study was supported by the National Nature Science Fund of China (Grant nos. 81471575 and 81273246).

\section{References}

[1] J. H. Chang and D. Wakefield, "Uveitis: a global perspective," Ocular Immunology and Inflammation, vol. 10, no. 4, pp. 263-279, 2002.

[2] J. H. Chang, P. J. McCluskey, and D. Wakefield, "Toll-like receptors in ocular immunity and the immunopathogenesis of inflammatory eye disease," The British Journal of Ophthalmology, vol. 90, no. 1, pp. 103-108, 2006.

[3] J. H. Chang, P. J. McCluskey, and D. Wakefield, "Acute anterior uveitis and HLA-B27," Survey of Ophthalmology, vol. 50, pp. 364-388, 2005.

[4] S. K. Biswas and E. Lopez-Collazo, "Endotoxin tolerance: new mechanisms, molecules and clinical significance," Trends in Immunology, vol. 30, no. 10, pp. 475-487, 2009.

[5] J. T. Rosenbaum, H. O. McDevitt, R. B. Guss, and P. R. Egbert, "Endotoxin-induced uveitis in rats as a model for human disease," Nature, vol. 286, no. 5773, pp. 611-613, 1980.

[6] A. F. De Vos, M. A. van Haren, C. Verhagen, R. Hoekzema, and A. Kijlstra, "Kinetics of intraocular tumor necrosis factor and interleukin- 6 in endotoxin-induced uveitis in the rat," Investigative Ophthalmology \& Visual Science, vol. 35, pp. 1100-1106, 1994.

[7] S. R. Planck, X. N. Huang, J. E. Robertson, and J. T. Rosenbaum, "Cytokine mRNA levels in rat ocular tissues after systemic endotoxin treatment," Investigative Ophthalmology \& Visual Science, vol. 35, pp. 924-930, 1994.

[8] M. Ojaniemi, V. Glumoff, K. Harju, M. Liljeroos, K. Vuori, and M. Hallman, "Phosphatidylinositol 3-kinase is involved in Toll-like receptor 4-mediated cytokine expression in mouse macrophages," European Journal of Immunology, vol. 33, no. 3, pp. 597-605, 2003.

[9] M. M. Monick, A. B. Carter, P. K. Robeff, D. M. Flaherty, M. W. Peterson, and G. W. Hunninghake, "Lipopolysaccharide activates Akt in human alveolar macrophages resulting in nuclear accumulation and transcriptional activity of betacatenin," Journal of Immunology, vol. 166, no. 7, pp. 47134720, 2001.

[10] B. W. Jones, K. A. Heldwein, T. K. Means, J. J. Saukkonen, and M. J. Fenton, "Differential roles of Toll-like receptors in the elicitation of proinflammatory responses by macrophages," Annals of the Rheumatic Diseases, vol. 60, Supplement 3, pp. iii6-iii12, 2001.

[11] Q. Yang, S. E. Calvano, S. F. Lowry, and I. P. Androulakis, "A dual negative regulation model of Toll-like receptor 4 signaling for endotoxin preconditioning in human endotoxemia," Mathematical Biosciences, vol. 232, no. 2, pp. 151-163, 2011. 
[12] X. F. Hu, H. Lu, J. Wang et al., "Screening of key genes and inflammatory signalling pathway involved in the pathogenesis of HLA-B27-associated acute anterior uveitis by gene expression microarray," Zhonghua Yan Ke Za Zhi, vol. 49, no. 3, pp. 217-223, 2013.

[13] W. Chen, X. Hu, L. Zhao, S. Li, and H. Lu, "Expression of tolllike receptor 4 in uvea-resident tissue macrophages during endotoxin-induced uveitis," Molecular Vision, vol. 15, pp. 619-628, 2009.

[14] Y. Endo, M. Shibazaki, K. Yamaguchi et al., "Enhancement by galactosamine of lipopolysaccharide(LPS)-induced tumour necrosis factor production and lethality: its suppression by LPS pretreatment," British Journal of Pharmacology, vol. 128, no. 1, pp. 5-12, 1999.

[15] R. Hoekzema, P. I. Murray, M. A. van Haren, M. Helle, and A. Kijlstra, "Analysis of interleukin-6 in endotoxin-induced uveitis," Investigative Ophthalmology \& Visual Science, vol. 32, no. 1, pp. 88-95, 1991.

[16] S. Yu, W. Zhang, J. Yu, S. Feng, T. Guo, and H. Lu, "Inhibiting effect of radix hedysari polysaccharide (HPS) on endotoxininduced uveitis in rats," International Immunopharmacology, vol. 21, no. 2, pp. 361-368, 2014.

[17] R. G. Tilton, K. Chang, J. A. Corbett et al., "Endotoxin-induced uveitis in the rat is attenuated by inhibition of nitric oxide production," Investigative Ophthalmology \& Visual Science, vol. 35, no. 8, pp. 3278-3288, 1994.

[18] D. C. Morrison and J. L. Ryan, "Endotoxins and disease mechanisms," Annual Review of Medicine, vol. 38, pp. 417-432, 1987.

[19] H. Mashimo, N. Ohguro, S. Nomura, N. Hashida, K. Nakai, and Y. Tano, "Neutrophil chemotaxis and local expression of interleukin-10 in the tolerance of endotoxin-induced uveitis," Investigative Ophthalmology \& Visual Science, vol. 49, no. 12, pp. 5450-5457, 2008.

[20] A. Lennikov, N. Kitaichi, K. Noda et al., "Amelioration of endotoxin-induced uveitis treated with an $\mathrm{I} \kappa \mathrm{B}$ kinase $\beta$ inhibitor in rats," Molecular Vision, vol. 18, pp. 2586-2597, 2012.

[21] A. Lennikov, N. Kitaichi, K. Noda et al., "Amelioration of endotoxin-induced uveitis treated with the sea urchin pigment echinochrome in rats," Molecular Vision, vol. 20, pp. 171-177, 2014, eCollection 2014.

[22] S. Keles, Z. Halici, H. T. Atmaca et al., "The ocular endothelin system: a novel target for the treatment of endotoxin-induced uveitis with bosentan," Investigative Ophthalmology \& Visual Science, vol. 55, no. 6, pp. 3517-3524, 2014.

[23] W. Zou, Z. Y. Li, C. L. Li, and Z. C. Cui, "Protein kinase B and its role in the signal transduction pathway mediated by phosphoinositide 3-kinase," Sheng li Ke Xue Jin Zhan, vol. 31, no. 2, pp. 120-124, 2000. 


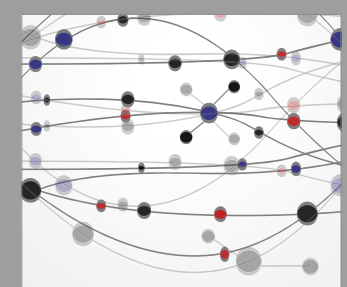

The Scientific World Journal
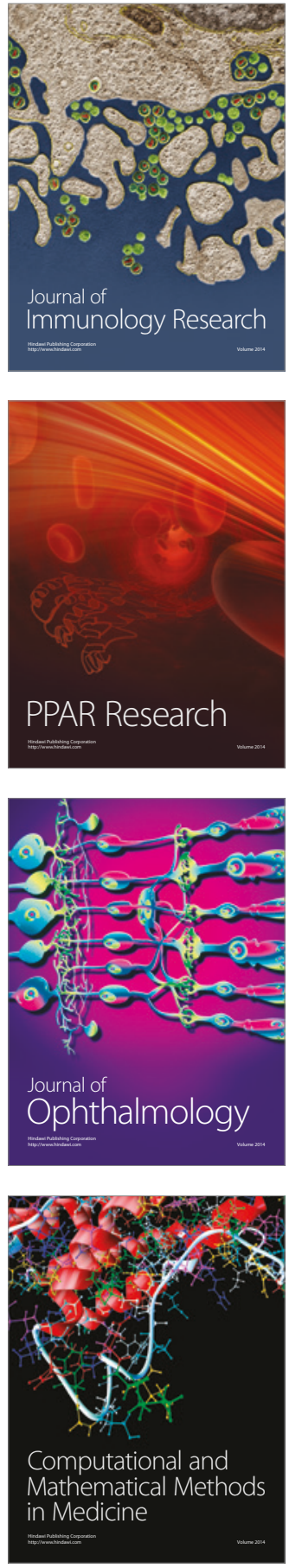

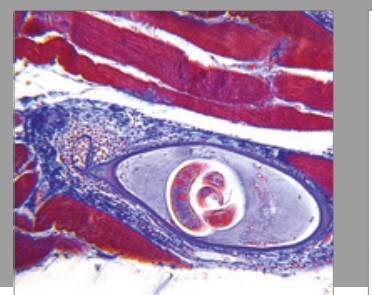

Gastroenterology Research and Practice
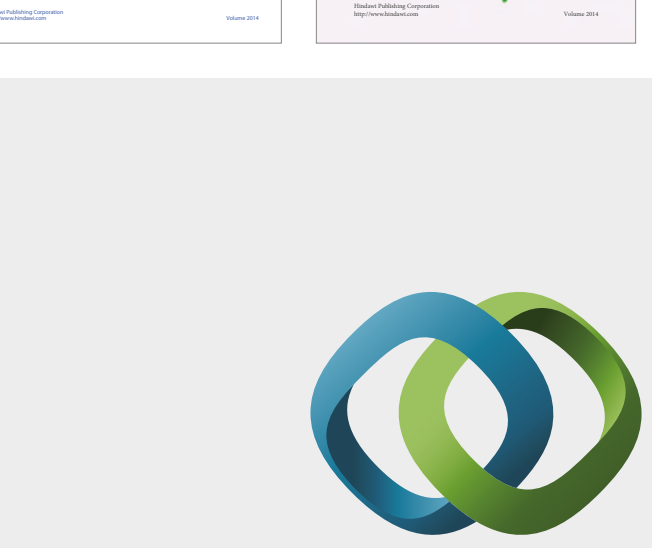

\section{Hindawi}

Submit your manuscripts at

https://www.hindawi.com
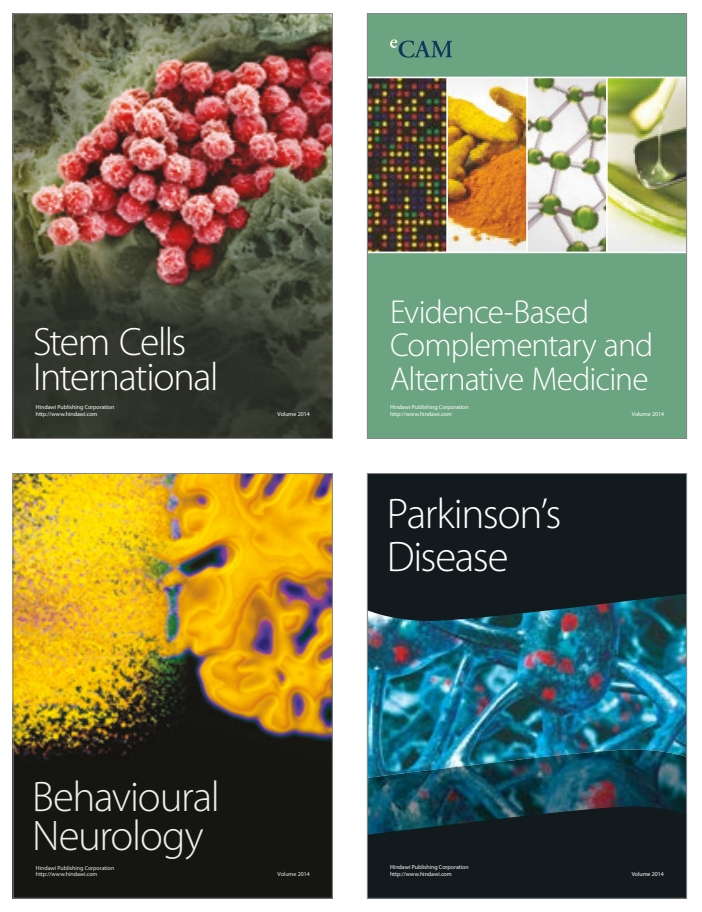
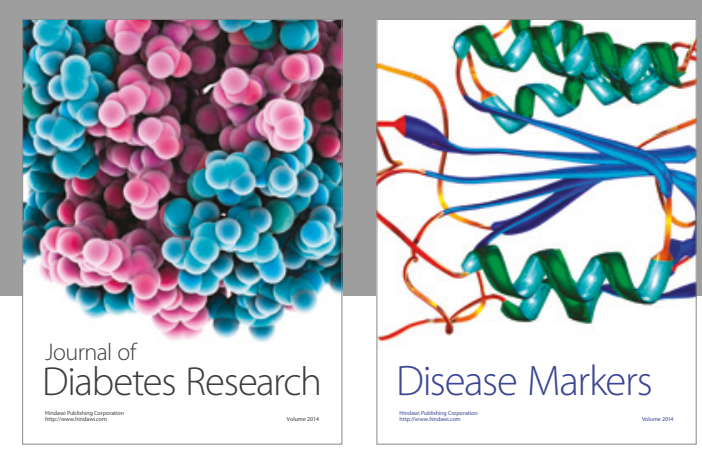

Disease Markers
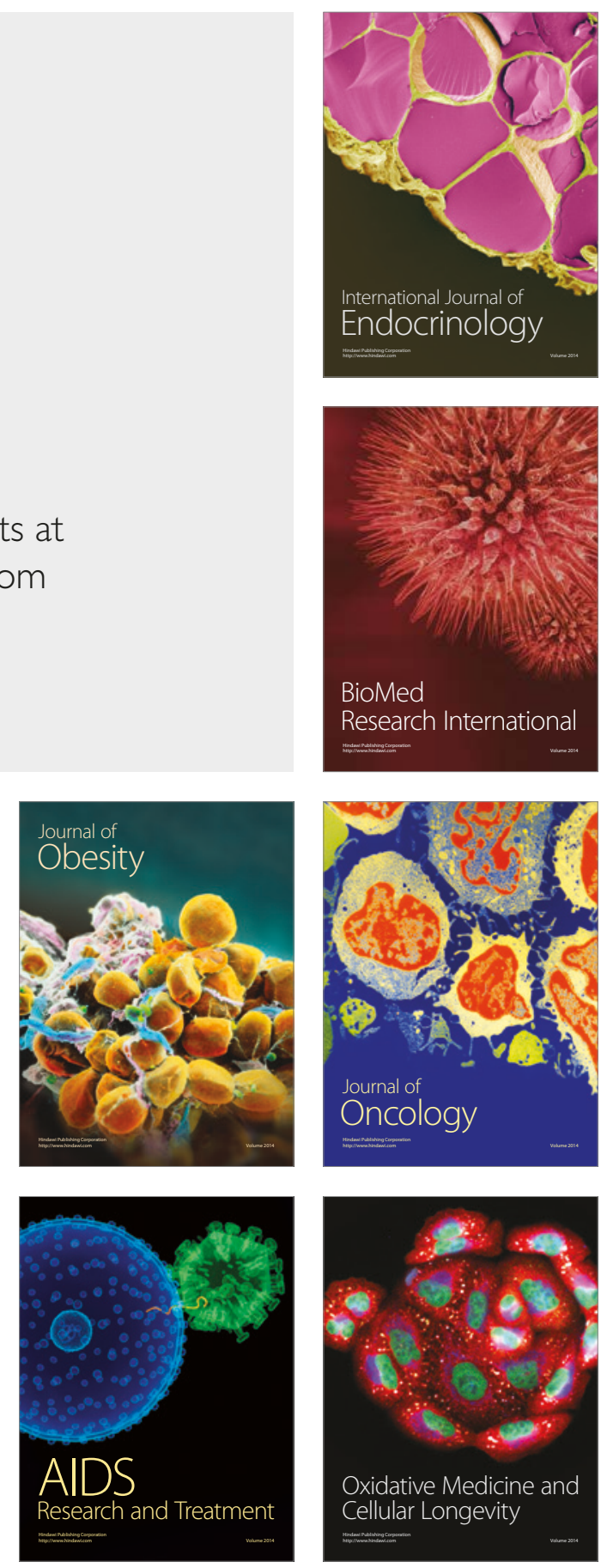